\title{
Methods and Tools for Ethical Usability
}

\author{
Iordanis Kavathatzopoulos ${ }^{1}$, Agata Kostrzewa ${ }^{2}$, and Mikael Laaksoharju ${ }^{1}$ \\ ${ }^{1}$ Uppsala University, Department of Information Technology - Human-Computer \\ Interaction, Box 337, 75105 Uppsala, Sweden \\ ${ }^{2}$ Uppsala University, Department of Business Studies, Box 513, 75120 Uppsala, Sweden \\ iordanis@it.uu.se, Agata.Kostrzewa@fek.uu.se, \\ Mikael.Laaksoharjulit.uu.se
}

\begin{abstract}
The objectives of the tutorial are to provide knowledge of basic ethical, psychological and organizational theories that are relevant to consider ethical aspects during design and use of IT systems; knowledge and skills about handling and solving ethical problems in connection with design and use of ITsystems; and skills in using questionnaires, surveys, interviews and the like in connection with software development and IT-use. It contains lectures, workshop and exercises; use of special tools to identify and consider IT ethical issues during planning, construction, installation and use of IT systems; and group exercises where the participants train their ethical skills on IT ethical conflicts and problems. Intended participants are system developers, purchasers, usability experts, academics, HCI teachers.
\end{abstract}

Keywords: Ethics, Usability, Tools, Methods, Skills.

\section{Ethical Competence for IT Systems}

Ethical problems caused by the use of IT may have very serious consequences. On the other hand, it is beyond any doubt that the anticipation of ethical problem situations and their proper solution can facilitate design, implementation and efficient use of IT.

Competence to handle ethical problems in a satisfying way is very important for any IT customer, designer or user. But these professionals, just as other people, are often lacking knowledge about what is the right thing to do in concrete IT systems construction situations. Applicable ethical guidelines are extremely difficult to formulate and therefore ethical guidelines are not any option. What would be possible though is to adopt a practical method to identify and solve ethical problems connected to IT use and design. Special tools and working methods can be applied to identify relevant values and stakeholder interests, and to integrate them into the design of the IT system under development.

This tutorial will offer the participants a basic knowledge of ethical and psychological decision-making theories as well as train skills to use certain tools and methods in handling ethical issues. The focus will not be on moral philosophical normative aspects but on individual and organizational ethical problem-solving and decisionmaking processes. The participants will be trained to use the tools and methods on representative cases as well as on own system development projects. 
There are many moral aspects to the introduction of new technology and there are different approaches to deal with these. Here we do not use normative theory. We only suggest tools and methods that can aid people, who are not informed about ethical theories, when making decisions related to ethical aspects. Our tools can increase the level of ethical competence before a decision is taken, by simply describing all relevant values and aspects for all involved parts in a structured way. By iteratively considering how each possible action or decision affects each possible value for each involved person, company, organisation, etc, a broader and more complex view of the moral dilemma is achieved. The output from these tools work both as a decision support, but also as a kind of documentation for future reference, for continuous dialog and for argumentation reasons. If someone later questions a decision then the documentation can work to explain it, or if additional aspects of the dilemma are revealed then the documentation can be extended with this.

The primary purpose is not to generate answers to particular problems, but rather to acquire a comprehensive view of the problem at hand and also to document the information on which one base the decision making. No matter what the final decision is, our method summarises all aspects considered during the decision-making process. A successful inclusion of moral aspects in IT systems analysis, decision making and decision application can easily fail. The cause of this failure may be found in the way thinking, problem solving and decision making are performed by, for example, a system developer. People use different ways to handle moral problems. Psychological theory and research differentiate between two different moral functions, heteronomy and autonomy, which decide a person's ability to handle moral problems.

Decision makers, developers, users and purchasers, need high ethical competence and confidence in working with ethical issues during IT systems development. That is necessary in order to solve moral problems, and to make moral decisions in accordance with relevant values, principles and interests. Spontaneous subjective reactions to moral issues may give solutions to problems, which probably satisfy one's moral feelings. However, with such more or less emotional reactions only, the relevant factors of the particular moral problem are certainly not fully taken into account. What is needed is a psychological approach to ethical competence and skills. It appears then that the process or the way one tries to identify relevant ethical aspects is what really matters. Suitable tools and working methods that support this way of handling ethical usability issues are necessary.

Our tools and methods, such as EthXpert, support the adoption of a holistic approach, of critical reasoning and of systematic analysis of the ethical aspects during concrete IT systems development. By using our tools and methods for the solution of IT ethical problems decision makers get a great amount of help to identify the significant problems, to make them explicit, and to reformulate them in order to be able to work with them. Decision makers, such as system developers or system purchasers, define their own (organizational as well as personal) position, duties, commitments, values and feelings. They also identify and take into consideration the interests, values and needs of all stakeholders. They generate alternative courses of action, and they systematically weigh each of them against all values and interests involved in the situation. By that they can consider all relevant ethical aspects and adopt the ethically most suitable design solutions. 